\title{
The Low-Temperature Structural Behavior of Sodium 1-Carba-closo-decaborate: $\mathrm{NaCB}_{9} \mathrm{H}_{10}$
}

Hui Wu ${ }^{* a}$ Wan Si Tang, ${ }^{a, b}$ Wei Zhou, ${ }^{a}$ Jacob D. Tarver, ${ }^{a, c}$ Vitalie Stavila, ${ }^{d}$ Craig M. Brown, ${ }^{a}$ and Terrence J. Udovic*a

${ }^{a}$ NIST Center for Neutron Research, National Institute of Standards and Technology, Gaithersburg, MD 20899-6102

${ }^{b}$ Department of Materials Science and Engineering, University of Maryland, College Park, MD 20742-2115

${ }^{c}$ National Renewable Energy Laboratory, Golden, CO 80401

${ }^{d}$ Energy Nanomaterials, Sandia National Laboratories, Livermore, CA 94551

Corresponding Authors: *E-mails: hui.wu@nist.gov; udovic@nist.gov

KEYWORDS: density functional theory, diffraction, ionic conductors, monocarba-closodecaborates, sodium borohydrides, vibrational spectroscopy

\footnotetext{
Abstract

Two ordered phases of the novel solid superionic conductor sodium 1-carba-closo-decaborate $\left(\mathrm{NaCB}_{9} \mathrm{H}_{10}\right)$ were identified via synchrotron x-ray powder diffraction in combination with firstprinciples calculations and neutron vibrational spectroscopy. A monoclinic packing of the large ellipsoidal $\mathrm{CB}_{9} \mathrm{H}_{10}{ }^{-}$anions prevails at the lowest temperatures, but a first-order transformation to a slightly modified orthorhombic packing is largely complete by $240 \mathrm{~K}$. The $\mathrm{CB}_{9} \mathrm{H}_{10}{ }^{-}$anion orientational alignments and $\mathrm{Na}^{+}$cation interstitial sitings in both phases are arranged so as to minimize the cation proximities to the uniquely more positive $\mathrm{C}$-bonded $\mathrm{H}$ atoms of the anions. These results provide valuable structural information pertinent to understanding the relatively low-temperature, entropy-driven, order-disorder phase transition for this compound.
} 


\section{Introduction}

Complex-hydride-based compounds such as $\mathrm{LiBH}_{4}$ and $\mathrm{Na}_{2} \mathrm{BH}_{4} \mathrm{NH}_{2}$ have been shown over the past decade to be capable of fast-ion conduction, suggesting that these types of materials may have potential as solid-state electrolytes [1,2]. In the last few years, novel salts comprised of $\mathrm{Li}^{+}$ and $\mathrm{Na}^{+}$cations and large polyhedral boron-hydrogen-based anions, such as $\mathrm{B}_{12} \mathrm{H}_{12}{ }^{2-}, \mathrm{B}_{10} \mathrm{H}_{10}{ }^{2-}$, $\mathrm{CB}_{11} \mathrm{H}_{12}{ }^{-}$, and $\mathrm{CB}_{9} \mathrm{H}_{10}{ }^{-}$, have emerged as particularly promising superionic electrolytes for nextgeneration, all-solid-state power devices $[3,4,5,6,7]$. Indeed, the most recently reported versions (with monovalent 1-carba-closo-decaborate $\mathrm{CB}_{9} \mathrm{H}_{10}{ }^{-}$anions), $\mathrm{LiCB}_{9} \mathrm{H}_{10}$ and $\mathrm{NaCB}_{9} \mathrm{H}_{10}$, in their disordered hexagonal phases exhibit liquid-like polycrystalline ionic conductivities of $0.03 \mathrm{~S}$ $\mathrm{cm}^{-1}$ at $354 \mathrm{~K}$ and $297 \mathrm{~K}$, respectively [6].

Although the structural details for the disordered phases of these two compounds are known [6], no definitive information has been reported, as of yet, concerning their lowtemperature ordered structures. The related $\mathrm{Li}_{2} \mathrm{~B}_{10} \mathrm{H}_{10}$ and $\mathrm{Na}_{2} \mathrm{~B}_{10} \mathrm{H}_{10}$ compounds with geometrically similar but divalently charged decahydro-closo-decaborate $\mathrm{B}_{10} \mathrm{H}_{10}{ }^{2-}$ anions are known to display ordered structures with respective hexagonal [8] and monoclinic symmetries $[9,10]$. Yet, one cannot assume that these same structural symmetries will apply to the ordered $\mathrm{LiCB}_{9} \mathrm{H}_{10}$ and $\mathrm{NaCB}_{9} \mathrm{H}_{10}$ phases, since one must take into account the structural consequences of the marked differences in anion valencies and charge polarizations between $\mathrm{CB}_{9} \mathrm{H}_{10}{ }^{-}$and $\mathrm{B}_{10} \mathrm{H}_{10}{ }^{2-}$. For example, although $\mathrm{Li}_{2} \mathrm{~B}_{12} \mathrm{H}_{12}$ and $\mathrm{Na}_{2} \mathrm{~B}_{12} \mathrm{H}_{12}$ with divalent dodecahydro-closo-dodecaborate $\mathrm{B}_{12} \mathrm{H}_{12}{ }^{2-}$ anions exhibit low-temperature ordered structures with respective cubic [11] and monoclinic symmetries [12], the related $\mathrm{LiCB}_{11} \mathrm{H}_{12}$ and $\mathrm{NaCB}_{11} \mathrm{H}_{12}$ compounds with monovalent monocarba-closo-decaborate $\mathrm{CB}_{11} \mathrm{H}_{12}{ }^{-}$anions both exhibit different ordered structures with orthorhombic symmetries [5]. 
In this paper, we focus on the structural behavior of (1-carba-) $\mathrm{NaCB}_{9} \mathrm{H}_{10}$. We have identified two low-temperature ordered structures for $\mathrm{NaCB}_{9} \mathrm{H}_{10}$ based on Rietveld model analysis of synchrotron x-ray powder diffraction (XRPD) data combined with first-principles calculations and corroborated by neutron vibrational spectroscopy (NVS).

\section{Experimental Details}

Sodium 1-carba-closo-decaborate $\left(\mathrm{NaCB}_{9} \mathrm{H}_{10}\right)$ was obtained from Katchem [13]. (N.B., as there are two possible monocarba- isomers, 1-carba- refers to carbon occupying an apical position; 2-carba- to carbon occupying one of the eight equatorial positions of the bicappedsquare-antiprismatic $\mathrm{CB}_{9} \mathrm{H}_{10}{ }^{-}$anion. Throughout this paper, it should be assumed that we are investigating the salt of the 1-carba- isomer form.) This highly hygroscopic salt was annealed under vacuum at $473 \mathrm{~K}$ for about $16 \mathrm{~h}$ to ensure full dehydration. It contained a minor $\mathrm{CB}_{11} \mathrm{H}_{12}{ }^{-}$ anion molar impurity of $3 \%$ and was investigated without further purification.

XRPD patterns of $\mathrm{NaCB}_{9} \mathrm{H}_{10}$ in a sealed quartz capillary were measured between $100 \mathrm{~K}$ and $298 \mathrm{~K}$ at the Advanced Photon Source on Beamline 17-BM-B at Argonne National Laboratory using a Si(111) monochromator $(\lambda=0.72768(1) \AA)$. Differential scanning calorimetry measurements between $150 \mathrm{~K}$ and $350 \mathrm{~K}$ were made with a Netzsch (STA 449 F1 Jupiter) TGA-DSC under He flow with Al sample pans. NVS measurements were performed at the National Institute of Standards and Technology Center for Neutron Research on the FilterAnalyzer Neutron Spectrometer (FANS) [14] using the $\mathrm{Cu}(220)$ monochromator with pre- and post-collimations of 20' of arc, yielding a full-width-at-half-maximum (fwhm) energy resolution of about $3 \%$ of the neutron energy transfer.

To assist the structural refinements, first-principles calculations were performed within the plane-wave implementation of the generalized gradient approximation to Density Functional 
Theory (DFT) using a Vanderbilt-type ultrasoft potential with Perdew-Burke-Ernzerhof exchange correlation [15]. A cutoff energy of $544 \mathrm{eV}$ and a $2 \times 2 \times 2 \mathrm{k}$-point mesh (generated using the Monkhorst-Pack scheme) were used and found to be enough for the total energy to converge within $0.01 \mathrm{meV} /$ atom. For comparison with the NVS measurements, the phonon density of states (PDOS) was calculated for the DFT-optimized $0 \mathrm{~K} \mathrm{NaCB}_{9} \mathrm{H}_{10}$ structure using the supercell method $(1 \times 2 \times 1$ cell size $)$ with finite displacements $[16,17]$ and were appropriately weighted to take into account the $\mathrm{H}, \mathrm{Na}, \mathrm{B}$, and $\mathrm{C}$ total neutron scattering cross sections. In addition, the PDOS of the isolated $1-\mathrm{CB}_{9} \mathrm{H}_{10}{ }^{-}$anion was calculated for comparison using a $30 \times 30 \times 30$ supercell and considering its $C_{4 \mathrm{v}}$ molecular symmetry. Lastly, DFT calculations of the isolated $2-\mathrm{CB}_{9} \mathrm{H}_{10}{ }^{-}$anion using a similar supercell construction were performed to estimate its relative stability.

All structural depictions were made using the VESTA (Visualization for Electronic and Structural Analysis) software [18]. For all figures, standard uncertainties are commensurate with the observed scatter in the data, if not explicitly designated by vertical error bars.

\section{Results and Discussion}

The $\mathrm{NaCB}_{9} \mathrm{H}_{10}$ capillary sample was cooled down to $100 \mathrm{~K}$ and held there for $1 \mathrm{~h}$ before collecting the initial XRPD pattern. Subsequently, the sample was heated to $298 \mathrm{~K}$ and a second XRPD pattern was collected. The resultant $100 \mathrm{~K}$ and $298 \mathrm{~K}$ patterns in Figs. 1 and 2 were indexed to respective monoclinic and orthorhombic structures. Assessment of the extinction symbols associated with the space groups of these two phases indicated the only two (highest) symmetries compatible with the formula volumes to be $P 2_{1} / c$ (No.14) at $100 \mathrm{~K}$ and $P n a 2_{1}$ (No.33) at $298 \mathrm{~K}$. The two different crystal structures were then solved using direct space 
methods under the corresponding space groups. Subsequently, DFT calculations were performed to optimize the $\mathrm{CB}_{9} \mathrm{H}_{10}{ }^{-}$rigid-body configurations and Na positions, followed by Rietveld refinement [19] of the DFT-relaxed structural models using the GSAS package [20]. Due to the relatively poor $\mathrm{X}$-ray sensitivity for $\mathrm{H}$, the optimized $\mathrm{CB}_{9} \mathrm{H}_{10}{ }^{-}$anion was kept as a rigid body during refinement. The thermal factors of like atoms were constrained to be the same. The coordinates of the $\mathrm{CB}_{9} \mathrm{H}_{10}{ }^{-}$anions and $\mathrm{Na}^{+}$cations together with the lattice parameters were refined, yielding the agreement factors of $R_{w p}=0.0352, R_{p}=0.0256$, and $\chi^{2}=3.21$ for the $100 \mathrm{~K}$ pattern and $\mathrm{R}_{\mathrm{wp}}=0.0402, \mathrm{R}_{\mathrm{p}}=0.0274$, and $\chi^{2}=3.79$ for the $298 \mathrm{~K}$ pattern. Refined lattice parameters were $a=14.3713(5) \AA, b=7.7224(2) \AA, c=19.6297(7) \AA$, and $\beta=132.608(1)^{\circ}$ for the $100 \mathrm{~K}$ monoclinic phase and $a=9.9830(3) \AA, b=10.6367(3) \AA$, and $c=7.8266(2) \AA$ for the $298 \mathrm{~K}$ orthorhombic phase. At $298 \mathrm{~K}$, a small fraction ( $\approx 3$ wt. \%) of normally higher-temperature disordered hexagonal phase [7] was also explicitly determined from refinement. Similarly, the $100 \mathrm{~K}$ pattern contains a number of rather small impurity-related peaks (such as at $7.06^{\circ}, 7.40^{\circ}$, and $7.58^{\circ}$ ), which we believe also reflect some disordered hexagonal phase (stabilized by incorporated $\mathrm{CB}_{11} \mathrm{H}_{12}{ }^{-}$impurity anions) and probably a small fraction of hydrate phases that are possible due to the extremely hygroscopic nature of this salt compound. Tables S1 and S2 summarizing the crystallographic details and the CIF files are included in the Supplementary Information (SI).

It should be noted that the monoclinic and orthorhombic structural differences are rather subtle, as reflected by the strong similarities in the XRPD patterns. The insets in Figs. 1 and 2 exemplify the main differences, i.e., that some of the Bragg peaks associated with the highersymmetry orthorhombic structure are actually narrowly split in the lower-symmetry monoclinic structure. 


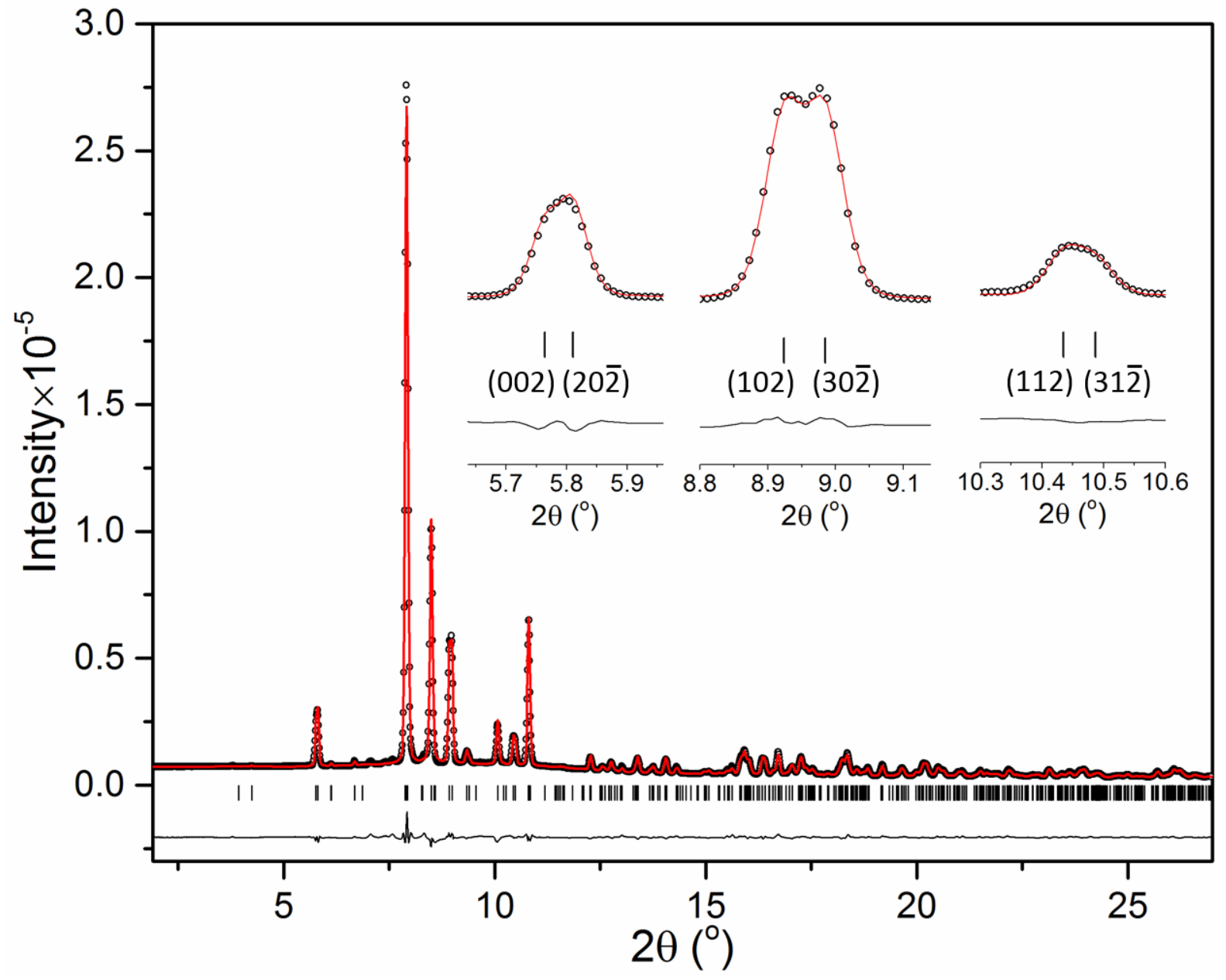

Figure 1. Experimental (circles), fitted (line), and difference (line below observed and calculated patterns) synchrotron XRPD profiles for $\mathrm{NaCB}_{9} \mathrm{H}_{10}$ at $100 \mathrm{~K}(\lambda=0.72768 \AA)$. Vertical bars indicate the calculated positions of the Bragg peaks. $R_{\mathrm{wp}}=0.0352, \mathrm{R}_{\mathrm{p}}=0.0256, \chi^{2}=3.21$. Insets show some characteristic peak splitting in this monoclinic structure in comparison with the orthorhombic phase shown in Fig. 2. Individual Bragg reflections are noted. 


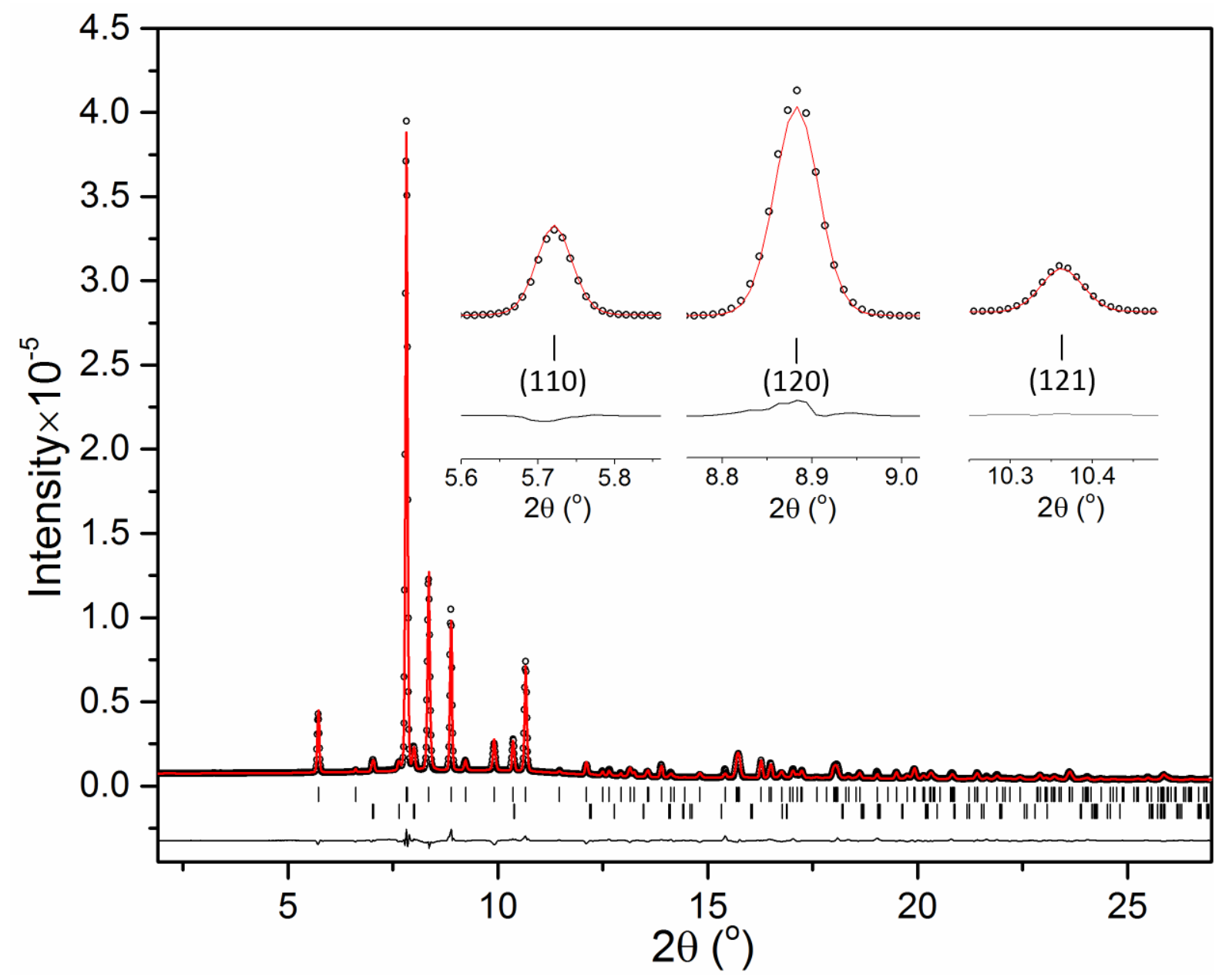

Figure 2. Experimental (circles), fitted (line), and difference (line below observed and calculated patterns) XRPD profiles for $\mathrm{NaCB}_{9} \mathrm{H}_{10}$ at $298 \mathrm{~K}(\lambda=0.72768 \AA$ ). Vertical bars indicate the calculated positions of Bragg peaks of the main ordered orthorhombic phase (97.01(7) wt. \%) and the normally higher-temperature disordered hexagonal phase [7] (2.99(5) wt. \%) (from the top). $\mathrm{R}_{\mathrm{wp}}=0.0402, \mathrm{R}_{\mathrm{p}}=0.0274, \chi^{2}=3.79$. Insets show the same characteristic peaks for this pattern as in that for the $100 \mathrm{~K}$ monoclinic structure but without the peak splitting evident in the latter. Individual Bragg reflections are noted. 

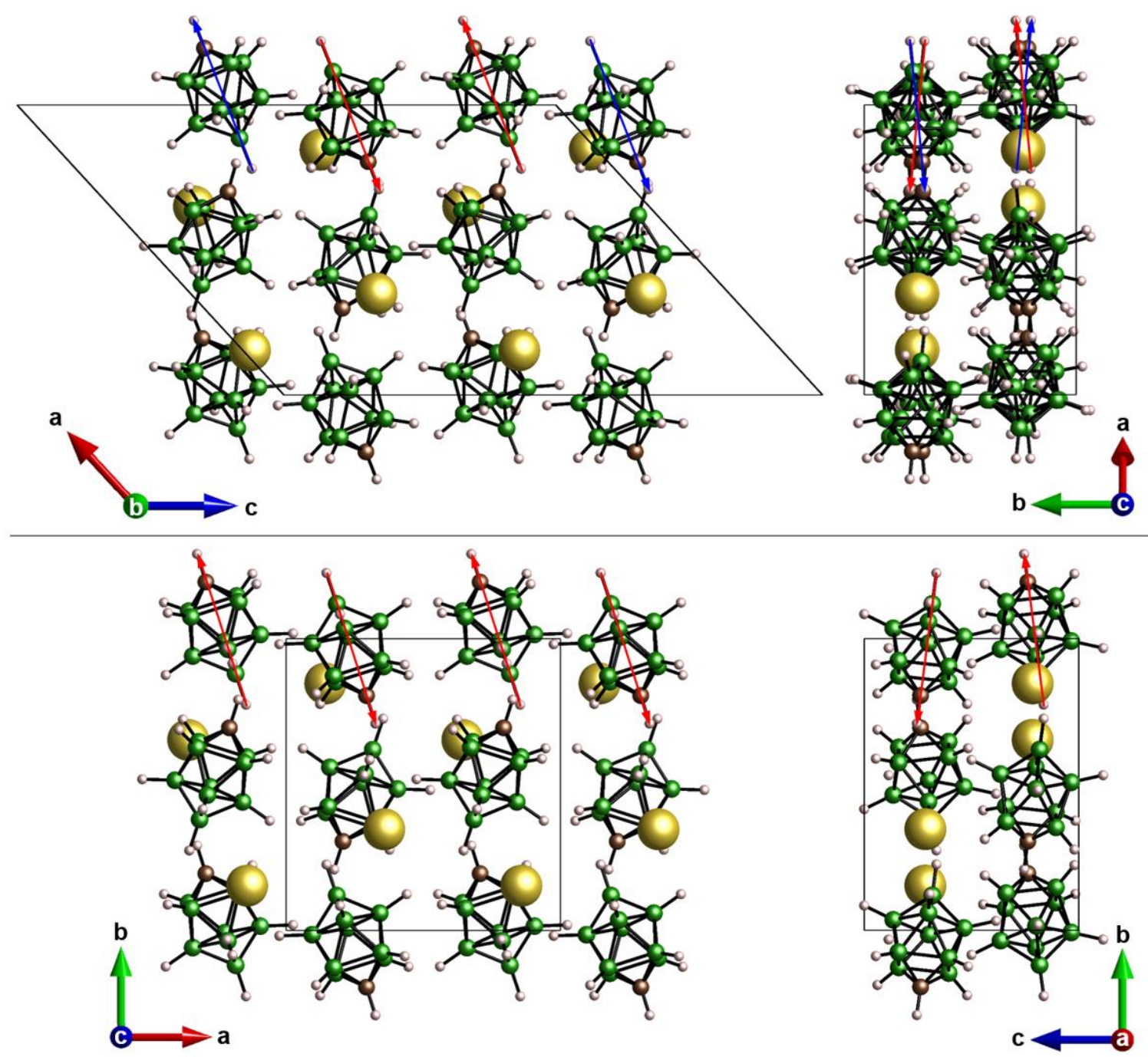

Figure 3. Crystal structures of the two ordered phases (top: lower-temperature $P 2_{1} / c$ monoclinic structure; bottom: higher-temperature $P n a 2_{1}$ orthorhombic structure). Arrows indicate the directions of $\mathrm{C}$ atoms in the $\mathrm{CB}_{9} \mathrm{H}_{10}{ }^{-}$anions. Red: outward plane (positive $\boldsymbol{b}$ direction); blue: inward plane (negative $\boldsymbol{b}$ direction).

Figure 3 compares the crystal structures of both phases, depicting these subtle differences. The red and blue arrows mark the alignments of the anion long axes in the direction of the $\mathrm{C}$ atoms, in effect, illustrating two sets of anion cantings (both red and blue) for the monoclinic structure, which merge into one set of cantings (red) for the orthorhombic structure. Moreover, as seen in 
Figs. 4 and 5, this translates into two slightly different $\mathrm{Na}^{+}$cation tetrahedral environments with respect to the anions for the monoclinic phase merging into only one $\mathrm{Na}^{+}$cation tetrahedral environment for the orthorhombic phase. In both structures, each $\mathrm{Na}^{+}$cation can be described as coordinated to two nearest-neighbor $\mathrm{H}$ atoms from each of the four surrounding anions, with $\mathrm{Na}$ H distances ranging from $2.28 \AA$ to $3.07 \AA$ (averaging $2.54 \AA$ ) for the monoclinic structure and from $2.25 \AA$ to $3.30 \AA$ (averaging $2.57 \AA$ ) for the orthorhombic structure. Moreover, distances from $\mathrm{Na}^{+}$to the anion geometric centers vary from $3.98 \AA$ to $4.41 \AA$ for the monoclinic structure (averaging 4.20 $\AA$ ) and from $4.10 \AA$ to $4.42 \AA$ (averaging $4.24 \AA$ ) for the orthorhombic structure. We note that all distances are estimated based on the DFT-optimized structures. Better accuracy would require more rigorous structure refinement using neutron powder diffraction data of deuterated compounds, where relaxation of the anion rigid-body constraint is justified.

As in the case for ordered orthorhombic $\mathrm{NaCB}_{11} \mathrm{H}_{12}$ [5], and as shown in Figs. 4 and 5, the $\mathrm{Na}^{+}$ cations favor positions that avoid close proximity to the more highly electropositive $\mathrm{C}$-bonded $\mathrm{H}$ atoms [6], so none of these $\mathrm{H}$ atoms are nearest neighbors of the $\mathrm{Na}^{+}$cations.

There are noteworthy similarities between the structural details of $\mathrm{NaCB}_{9} \mathrm{H}_{10}$ and $\mathrm{Na}_{2} \mathrm{~B}_{10} \mathrm{H}_{10}$, the latter which is comprised of geometrically similar building blocks. Although $\mathrm{Na}_{2} \mathrm{~B}_{10} \mathrm{H}_{10}$ possesses divalent as opposed to monovalent anions and twice as many cations per formula unit as $\mathrm{NaCB}_{9} \mathrm{H}_{10}$, it also displays monoclinic $P 2_{1} / c$ symmetry at low temperatures [10]. Moreover, each $\mathrm{Na}^{+}$cation is also most closely coordinated to two $\mathrm{H}$ atoms from each of four surrounding anions, with similar ranges of $\mathrm{Na}-\mathrm{H}$ and $\mathrm{Na}$-anion distances as observed for $\mathrm{NaCB}_{9} \mathrm{H}_{10}$.

Nonetheless, there is no indication of an order-order transition for $\mathrm{Na}_{2} \mathrm{~B}_{10} \mathrm{H}_{10}$, as for $\mathrm{NaCB}_{9} \mathrm{H}_{10}$. Such a transition may be a consequence of the different distributions of charge on the $\mathrm{H}$ atoms of the $\mathrm{CB}_{9} \mathrm{H}_{10}{ }^{-}$and $\mathrm{B}_{10} \mathrm{H}_{10}{ }^{2-}$ anions, although further insight probably requires theoretical analysis. 




Figure 4. Tetrahedral coordinations of the two crystallographically distinct $\mathrm{Na}^{+}$cations in the $P 2{ }_{1} / c$ monoclinic structure refined using rigid-body anions fixed to DFT-optimized values. The distances between $\mathrm{Na}^{+}$and the nearest $\mathrm{H}$ atoms, and between $\mathrm{Na}^{+}$and the geometric centers of the nearest $\mathrm{CB}_{9} \mathrm{D}_{10}{ }^{-}$anions are indicated. 




Figure 5. Tetrahedral coordination of the $\mathrm{Na}^{+}$cation (only one type crystallographic site) in the Pna $2_{1}$ orthorhombic structure refined using rigid-body anions fixed to DFT-optimized values. The distances between $\mathrm{Na}^{+}$and the nearest $\mathrm{H}$ atoms, and between $\mathrm{Na}^{+}$and the geometric centers of the nearest $\mathrm{CB}_{9} \mathrm{D}_{10}{ }^{-}$anions are indicated.

In order to determine where the monoclinic-orthorhombic transition actually occurs, we performed additional XRPD measurements between $163 \mathrm{~K}$ and $292 \mathrm{~K}$ and followed the progression of the Bragg peak located between $8.35^{\circ}$ and $8.47^{\circ} 2 \theta$ corresponding to the $(20-4)$ monoclinic-phase and related (200) orthorhombic-phase reflections (see Fig. 6). Analysis of this Bragg peak vs. temperature indicated that it was comprised of the reflections from both phases within the $210 \mathrm{~K}$ to $240 \mathrm{~K}$ transition region, signaling a first-order type transition. Figure 6a clearly shows the dogleg progression in the peak position, signaling the small step change in the related $d$-spacing associated with these reflections. The sequence of intensity vs. $2 \theta$ plots with temperature for this Bragg peak is shown in Fig. S1 of the SI. Deconvolution of the two 
components allowed us to plot the intensity fraction of each component Bragg reflection vs. temperature in Fig. $6 \mathrm{~b}$. We note that this material is found to be thermodynamically "sluggish" upon cooling, i.e., a period of annealing time is required after cooling down below the phasetransition region to transform the higher-temperature orthorhombic phase completely to the monoclinic phase. A relatively short annealing time of around $15 \mathrm{~min}$ at $100 \mathrm{~K}$ before collecting the data in Fig. 6 is why there was still about $12 \%$ of the orthorhombic phase present at $163 \mathrm{~K}$ prior to warming up the sample. No further attempt was made to measure the extent of this sluggishness or to determine any possible changes in the width of the transition region due to

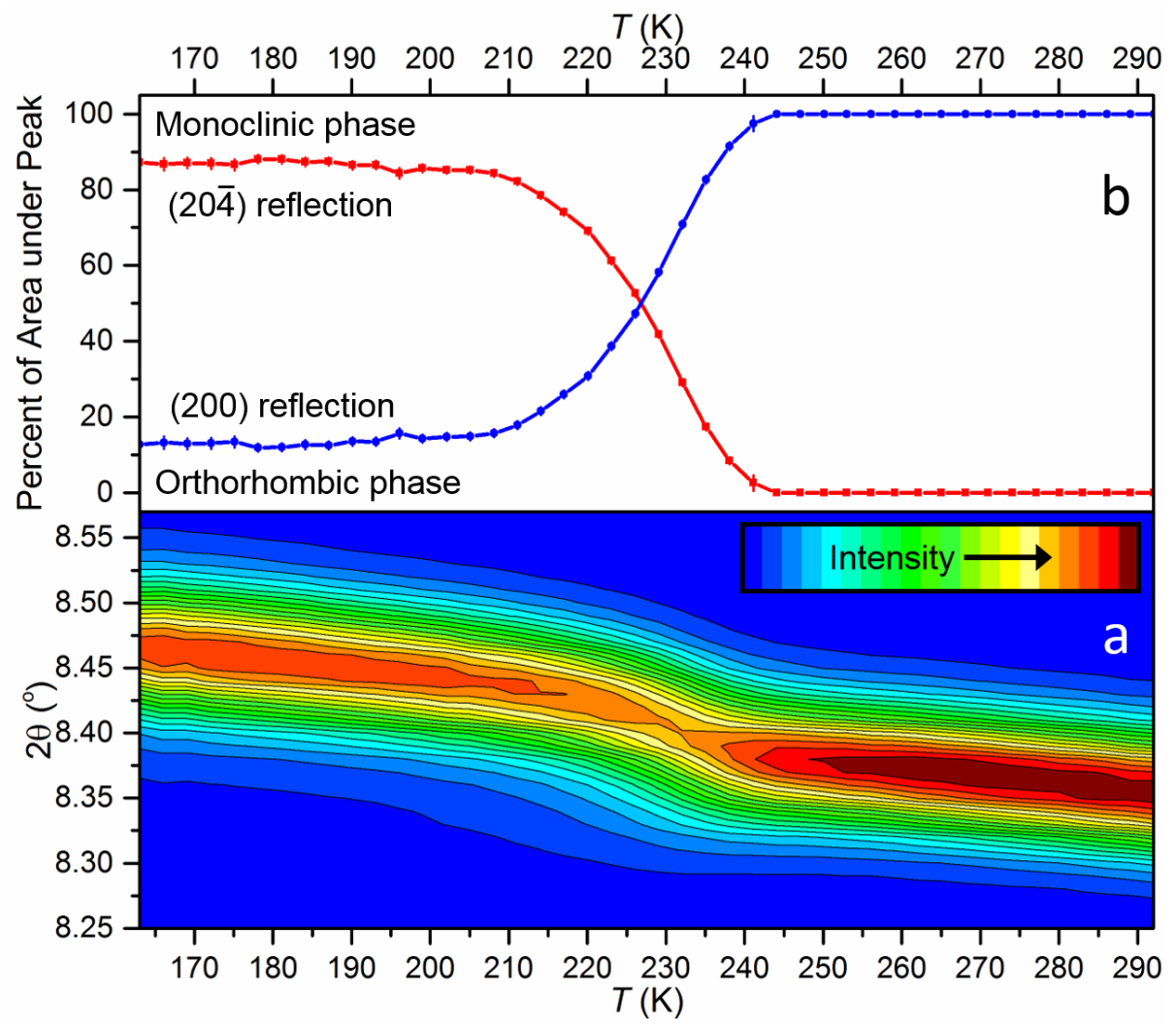

changes in the temperature ramp rate.

Figure 6. (a) Topographic plot showing the progression of the $\mathrm{NaCB}_{9} \mathrm{H}_{10}$ Bragg peak intensities corresponding to the (20-4) monoclinic-phase and (200) orthorhombic-phase reflections with $2 \theta$ upon heating $6 \mathrm{~K} \mathrm{~min}^{-1}$ from $163 \mathrm{~K}$ to $292 \mathrm{~K}$, and (b) corresponding fractional integrated areas of 
each Bragg reflection vs. temperature. 
Figure 7 shows the DSC heating scan from $150 \mathrm{~K}$ to $350 \mathrm{~K}$ after cooling the $\mathrm{NaCB}_{9} \mathrm{H}_{10}$ sample down to near $100 \mathrm{~K}$ and holding there for about $15 \mathrm{~min}$. Despite using a large sample (14 mg) and ramp rate $\left(20 \mathrm{~K} \mathrm{~min}^{-1}\right)$, there is no obvious endothermic feature in the expected monoclinicorthorhombic transition region ( $210 \mathrm{~K}$ to $240 \mathrm{~K}$ ), although the expected double-peak structure at higher temperatures signaling the order-disorder transition [6] is clearly evident. This suggests that the enthalpy change between the monoclinic and orthorhombic phases is extremely small.

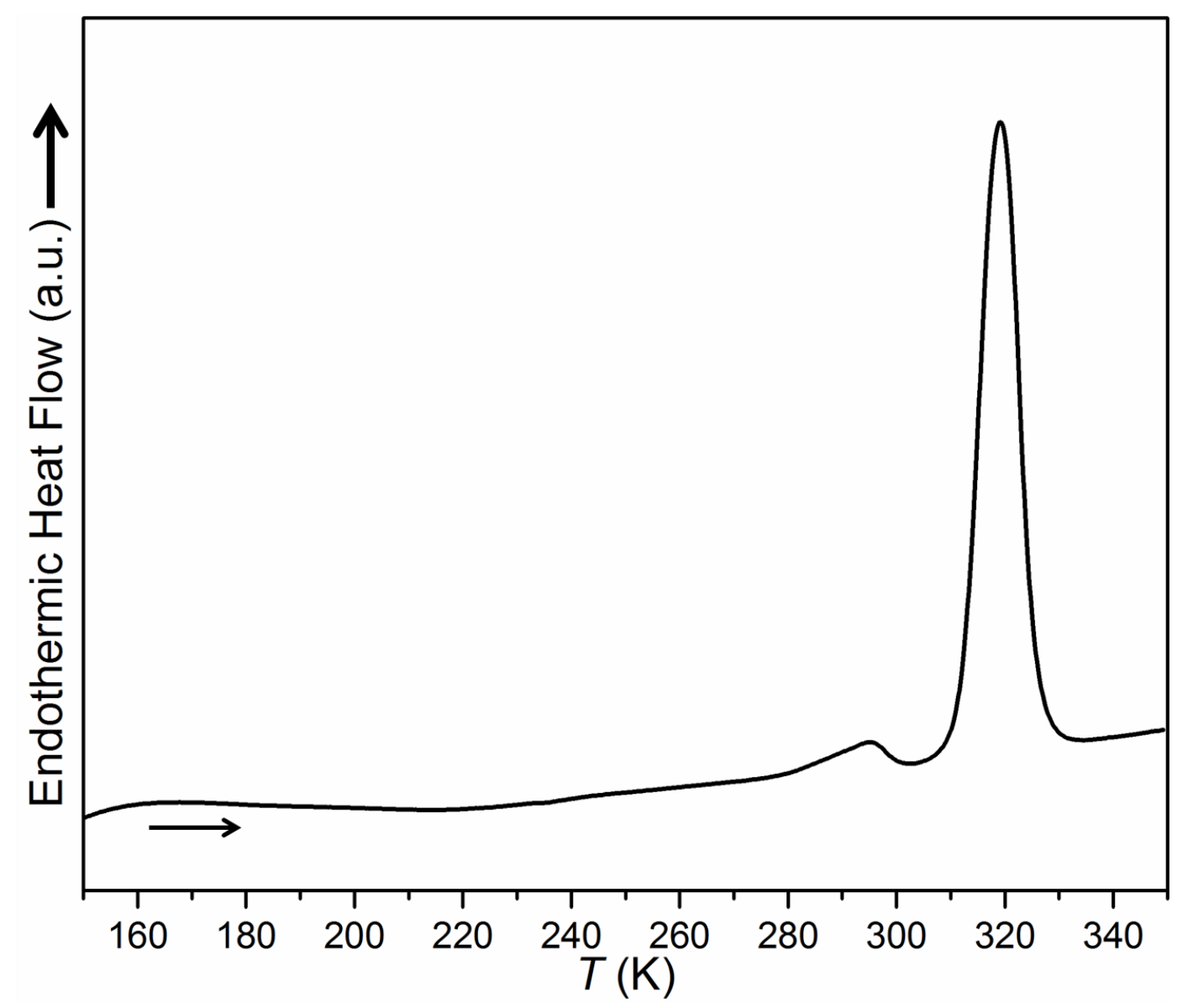

Figure 7. DSC heating scan $\left(20 \mathrm{~K} \mathrm{~min}^{-1}\right)$ for $\mathrm{NaCB}_{9} \mathrm{H}_{10}$ after cooling and maintaining near $100 \mathrm{~K}$ for about $15 \mathrm{~min}$. 


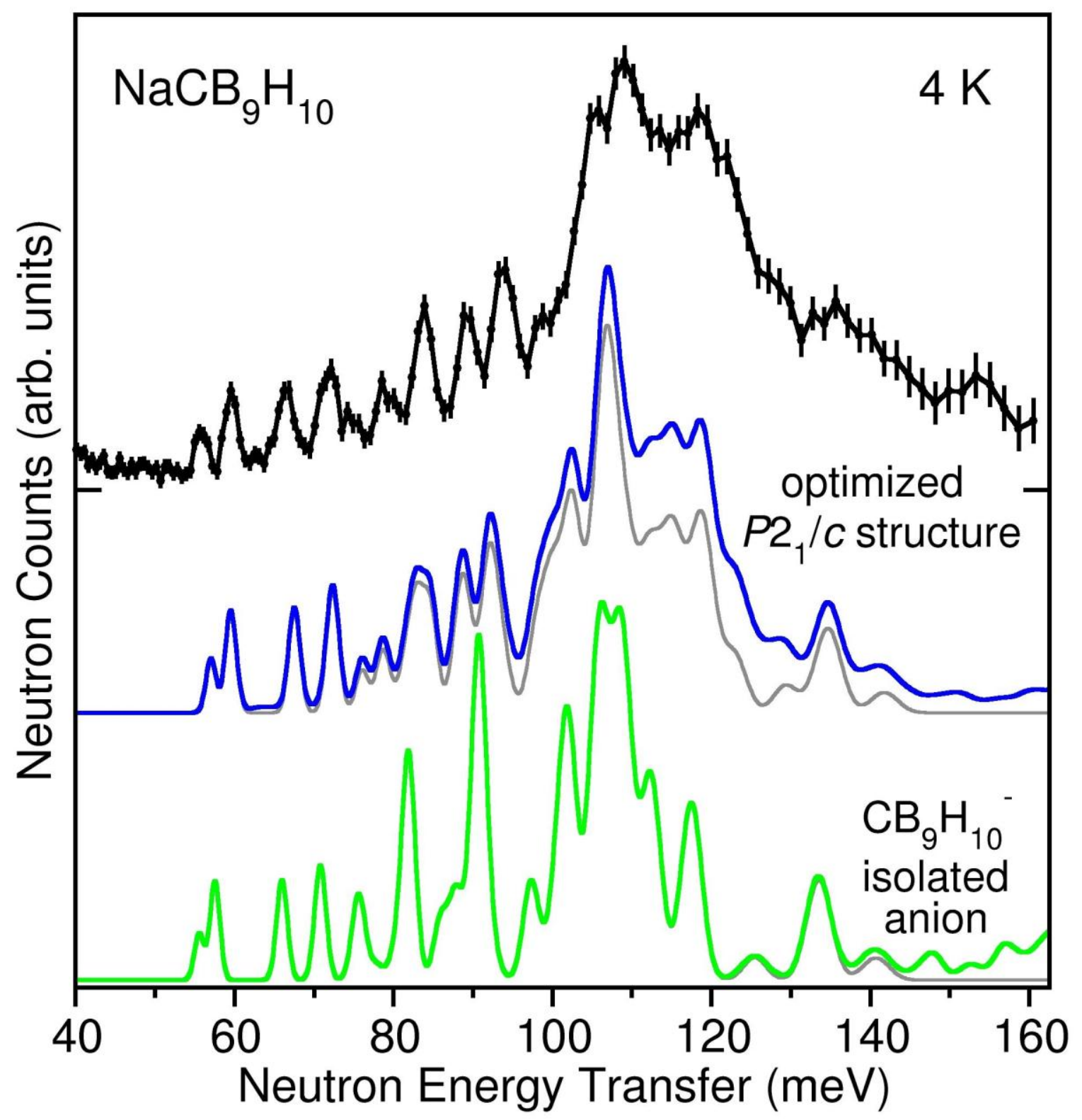

Figure 8. Neutron vibrational spectra (black) of $\mathrm{NaCB}_{9} \mathrm{H}_{10}$ at $4 \mathrm{~K}$ compared to the simulated one-phonon (gray) and one+two-phonon densities of states from first-principles phonon calculations of the optimized monoclinic $P 2_{1} / c$ structure (blue) and the isolated $\mathrm{CB}_{9} \mathrm{H}_{10}{ }^{-}$anion (green). (N.B., $1 \mathrm{meV} \approx 8.066 \mathrm{~cm}^{-1}$.)

The neutron vibrational spectrum for $\mathrm{NaCB}_{9} \mathrm{H}_{10}$ at $4 \mathrm{~K}$ is shown in Fig. 8 and compared with the simulated PDOSs of both the DFT-optimized $P 2_{1} / c$ structure and the isolated $\mathrm{CB}_{9} \mathrm{H}_{10}{ }^{-}$anion. 
In general, the PDOSs for closo-borate compounds, especially for the higher-energy modes, are known to be sensitive to the structural details $[5,8,10,21]$. This is also inferred by the observed differences in the calculated PDOSs between $75 \mathrm{meV}$ and $125 \mathrm{meV}$ for monoclinic $\mathrm{NaCB}_{9} \mathrm{H}_{10}$ and the isolated $\mathrm{CB}_{9} \mathrm{H}_{10}{ }^{-}$anion. The overall good agreement between the experimental and calculated PDOSs for $\mathrm{NaCB}_{9} \mathrm{H}_{10}$ further corroborates the low-temperature monoclinic structure determined by XRPD. Further information about the characters and energies of the different phonon modes contributing to the simulated PDOSs can be found in the SI.

The temperature-dependent structural details of this $\mathrm{NaCB}_{9} \mathrm{H}_{10}$ compound are interesting both fundamentally and technologically speaking. It would be interesting to compare further the structural behavior of this sodium salt with that of the 2-carba- isomer. Although thermodynamically less favored [22], (our isolated-anion DFT calculations at $0 \mathrm{~K}$ indicate it is more unstable than the 1-carba- isomer by $\sim 812 \mathrm{meV}$ per anion) this version, if it could be synthesized in pure form, might have a relatively lower order-disorder transition temperature due to its relatively lower anion symmetry. The exact temperature will depend on the relative enthalpies of its ordered and disordered phases compared to those associated with its isomeric cousin.

\section{Conclusion}

Two ordered structures of $\mathrm{NaCB}_{9} \mathrm{H}_{10}$ were determined by X-ray powder diffraction in combination with first-principles computations and corroborated by neutron vibrational spectroscopy: a lower-temperature monoclinic phase that fully transforms to a structurally similar orthorhombic phase by around $240 \mathrm{~K}$. These results provide a more complete picture of the $\mathrm{NaCB}_{9} \mathrm{H}_{10}$ temperature-dependent structural behavior. Such information will aid in 
developing a better theoretical understanding of the dramatic transformation to superionic conduction that occurs in this and the other various related closo-borate-type salt compounds.

\section{Acknowledgments}

Sandia National Laboratories is a multi-program laboratory managed and operated by Sandia Corporation, a wholly owned subsidiary of Lockheed Martin Corporation, for the US DOE's National Nuclear Security Administration under contract DE-AC04-94AL85000. J. T. gratefully acknowledges research support from the US DOE Office of Energy Efficiency and Renewable Energy, Fuel Cell Technologies Office, under Contract No. DE-AC36-08GO28308. Use of the Advanced Photon Source, an Office of Science User Facility operated for the US DOE Office of

Science by Argonne National Laboratory, was supported by the US DOE under Contract No. DE-AC02-06CH11357.

\section{References}

[1] A. Unemoto, M. Matsuo, S. Orimo, Complex Hydrides for Electrochemical Energy Storage. Adv. Funct. Mater. 24 (2014) 2267-2279. 
[2] P. E. de Jongh, D. Blanchard, M. Matsuo, T.J. Udovic, S. Orimo, Complex Hydrides as Room Temperature Solid Electrolytes for Rechargeable Batteries, Appl. Phys. A 122 (2016) 251.

[3] T. J. Udovic, M. Matsuo, A. Unemoto, N. Verdal, V. Stavila, A. V. Skripov, J. J. Rush, H. Takamura, S. Orimo, Sodium Superionic Conduction in $\mathrm{Na}_{2} \mathrm{~B}_{12} \mathrm{H}_{12}$, Chem. Commun. 50 (2014) 3750-3752.

[4] T. J. Udovic, M. Matsuo, W. S. Tang, H. Wu, V. Stavila, A. V. Soloninin, R. V. Skoryunov, O. A. Babanova, A. V. Skripov, J. J. Rush, A. Unemoto, H. Takamura, S. Orimo, Exceptional Superionic Conductivity in Disordered Sodium Decahydro-closoDecaborate, Adv. Mater. 26 (2014) 7622-7626.

[5] W. S. Tang, A. Unemoto, W. Zhou, V. Stavila, M. Matsuo, H. Wu, S. Orimo, T. J. Udovic, Unparalleled Lithium and Sodium Superionic Conduction in Solid Electrolytes with Large Monovalent Cage-like Anions, Energy Environ. Sci. 8 (2015) 3637-3645.

[6] W. S. Tang, M. Matsuo, H. Wu, V. Stavila, W. Zhou, A. A. Talin, A. V. Soloninin, R. V. Skoryunov, O. A. Babanova, A. V. Skripov, A. Unemoto, S. Orimo, T. J. Udovic, Liquidlike Ionic Conduction in Solid Lithium and Sodium Monocarba-closo-decaborates near or at Room Temperature, Adv. Energy Mater. 6 (2016) 1502237.

[7] W. S. Tang, M. Matsuo, H. Wu, V. Stavila, A. Unemoto, S. Orimo, T. J. Udovic, Stabilizing Lithium and Sodium Fast-Ion Conduction in Solid Polyhedral-Borate Salts at Device-Relevant Temperatures, Energy Storage Mater. 4 (2016) 79-83. 
[8] H. Wu, W. S. Tang, V. Stavila, W. Zhou, J. J. Rush, T. J. Udovic, The Structural Behavior of $\mathrm{Li}_{2} \mathrm{~B}_{10} \mathrm{H}_{10}$, J. Phys. Chem. C 119 (2015) 6481-6487.

[9] K. Hofmann, B. Albert, Crystal Structures of $\mathrm{M}_{2}\left[\mathrm{~B}_{10} \mathrm{H}_{10}\right](\mathrm{M}=\mathrm{Na}, \mathrm{K}, \mathrm{Rb})$ via Real-Space Simulated Annealing Powder Techniques, Z. Kristallogr. 220 (2005) 142-146.

[10] H. Wu, W. S. Tang, W. Zhou, V. Stavila, J. J. Rush, T. J. Udovic, The Structure of Monoclinic $\mathrm{Na}_{2} \mathrm{~B}_{10} \mathrm{H}_{10}$ : A Combined Diffraction, Spectroscopy, and Theoretical Approach, CrystEngComm 17 (2015) 3533-3540.

[11] J.-H. Her, M. Yousufuddin, W. Zhou, S. S. Jalisatgi, J. G. Kulleck, J. A. Zan, S.-J. Hwang, R. C. Bowman, Jr., T. J. Udovic, Crystal Structure of $\mathrm{Li}_{2} \mathrm{~B}_{12} \mathrm{H}_{12}$ : a Possible Intermediate Species in the Decomposition of $\mathrm{LiBH}_{4}$, Inorg. Chem. 47 (2008) 9757-9759.

[12] J.-H. Her, W. Zhou, V. Stavila, C. M. Brown, T. J. Udovic, Role of Cation Size on the Structural Behavior of the Alkali-Metal Dodecahydro-closo-Dodecaborates, J. Phys. Chem. C 113 (2009) 11187-11189.

[13] The mention of all commercial suppliers in this paper is for clarity and does not imply the recommendation or endorsement of these suppliers by NIST.

[14] T. J. Udovic, C. M. Brown, J. B. Leão, P. C. Brand, R. D. Jiggetts, R. Zeitoun, T. A. Pierce, I. Peral, J. R. D. Copley, Q. Huang, D. A. Neumann, R. J. Fields, The Design of a Bismuth-based Auxiliary Filter for the Removal of Spurious Background Scattering Associated with Filter-Analyzer Neutron Spectrometers, Nucl. Instr. and Meth. A 588 (2008) 406-413. 
[15] Quantum-ESPRESSO package: www.pwscf.org.

[16] G. Kresse, J. Furthmuller, J. Hafner, Ab initio Force Constant Approach to Phonon Dispersion Relations of Diamond and Graphite, Europhys. Lett. 32 (1995) 729-734.

[17] T. Yildirim, Structure and Dynamics from Combined Neutron Scattering and FirstPrinciples Studies, Chem. Phys. 261 (2000) 205-216.

[18] K. Momma, F. Izumi, VESTA 3 for Three-Dimensional Visualization of Crystal, Volumetric and Morphology Data, J. Appl. Crystallogr. 44 (2011) 1272-1276.

[19] H. M. Rietveld, A Profile Refinement Method for Nuclear and Magnetic Structures, J. Appl. Crystallogr. 2 (1969) 65-71.

[20] A. C. Larson, R. B. Von Dreele, General Structure Analysis System, Report LAUR 86-748; Los Alamos National Laboratory, NM, 1994.

[21] N. Verdal, W. Zhou, V. Stavila, J.-H. Her, M. Yousufuddin, T. Yildirim, T. J. Udovic, Alkali and Alkaline-Earth Metal Dodecahydro-closo-Dodecaborates: Probing Structural Variations via Neutron Vibrational Spectroscopy, J. Alloys Compds. 509S (2011) S694-S697.

[22] E. L Muetterties, W. H. Knoth, Polyhedral Boranes, Marcel Dekker, Inc., New York, 1968. 


\section{Graphical Abstract}

Ordered monoclinic and orthorhombic $\mathrm{NaCB}_{9} \mathrm{H}_{10}$ phases were determined by XRD and DFT computations and corroborated by neutron vibrational spectroscopy.



